\title{
Stage and fractality of ecogeosystems
}

\section{Opinion}

Ecogeosystems are hierarchies of interrelated geo-bio-and anthroposystems in which each succeeding member, younger and more complex, appeared and develops at the expense of the substance and energy of the previous ones, inheriting a certain commonality of signs and behavior. An ecogeosystem can be represented as a set of flexible bonds of particles (subsystems) that possess the energy that it exchanges with its environment.

Exchange is accompanied by deformation of bonds and particle motions. Abstracting from the nature of particles and the mechanisms of their interaction, the evolution of ecogeosystems can be interpreted generally as the accumulation of deformations. Such an approach is convenient in that the general patterns of development are revealed from experiments with simple models that can be quantitatively analyzed, for example, with a loaded solid body.

The analysis showed that the deformation of the loaded body, like the development of all ecogeosystems, includes three distinct stages: formation (childhood), maturity, degradation (old age), and one hidden, in different contexts called the incubation or embryonic period, pre-life, prehistory .. For generalization, it is advisable to express the deformation in a dimensionless form: $\mathrm{j}=(\mathrm{hn}-\mathrm{h}) /(\mathrm{hn}-\mathrm{he})$, where $\mathrm{hn}$ and $\mathrm{h}$ is the initial and current characteristic size of the body, he is the size of the elementary particle (in three-dimensional problems it is volume). The strain rate decreases at the 1st stage, is constant at the $2 \mathrm{nd}$ and increases at the $3 \mathrm{rd}$. The value of $\mathrm{j}$ consists of elastic, plastic, and tensile deformations. The total and the last two increase at all stages. Elastic, prevailing at the 1st stage, first increases to a maximum - the "vital" energy increases, and then, at the $2^{\text {nd }}$ stage, decreases to a minimum, followed by a degradation stage with a predominance of discontinuous deformations. Further development goes into a hidden stage. From the beginning and the end of the trajectory, on one side, there is an area of reality, determinism. On the other, invisible - the field of irrationalism, the "looking-glass", where things and time lose their certainty, nature as if is sleeping. Therefore, to the 3rd explicit developmental stages (in the life of the Earth, by the phanerozoic), the 4th implicit (cryptosis) is added. In the annual and daily cycles, spring, summer, autumn and morning, day, evening - explicit stages, winter and night — are implicit. In the life of the earth, cryptose - Precambrian takes about 4billion years, about $90 \%$ of its entire history. It speaks about the youth of the Earth - the share of the "dark" area in its annual, daily and monthly cycles is on average $25 \%$. At the latent stage, the cycle closes, from the old a new one is born - the system breaks up into parts (offspring) in which the initial conditions are restored in a leap. Therefore, in subsequent cycles, with the same load, the descendants behave like the "parent" system, deforming and splitting into smaller and smaller parts.

Elementary time $\tau$ e corresponds to an elementary (minimum possible with a given accuracy of observations) dimensional $-\Delta$ he or dimensionless - Ee deformation, with $\Delta$ he he. With the development of science, $\tau$ e and je tend to their maximum minima. In general, the number of elementary bonds (and deformations) is one less than that of elementary particles. In real systems, the number of particles in which is very large, the unit can be neglected.
Volume 3 Issue 5 - 2019

\author{
Konovalov AA \\ Doctor of Engineering Sciences, Chief Research Officer of \\ the Tyumen Scientific Center, Siberian Branch of the Russian \\ Academy of Sciences, Full Professor of Tyumen Industrial \\ University, Russia
}

Correspondence: Konovalov AA, Doctor of Engineering Sciences, Chief Research Officer of the Tyumen Scientific Center, Siberian Branch of the Russian Academy of Sciences, Full Professor of Tyumen Industrial University, Russia, Email konov7@rambler.ru

Received: July 15, 2019 | Published: September 16, 2019

Ecogeosystems are fractal space-time sets expressed by the sum of single (elementary) particles to the power $\mathrm{n}$. If $\mathrm{n}$ is an integer, then we have a self-similarity that determines the linear or reducing laws of evolution and hierarchies of ecogeosystems. If fractional, the system is non-linear. Ecogeosystems, starting with the "primordial": Sun-Earth, are close to self-similarity, since the way of their existence is identical: vibrationally - rotational, cyclic, and the cycles are composite: smaller, Derivatives from large, as if "embedded" (integrated) in them. But this semblance is incomplete, inherent only in the $2^{\text {nd }}$, linear stage of development. In general, it is not possible to find such an element of the ecogeosystem that would enter it an integer number of times. For example, a year is not divided without remainder, neither by month, nor by day, nor into smaller parts.

The stronger and longer the "pressure" of the medium, the greater the zone of its influence, the greater the deformation and the less elementary (non-deformable) part of the system. Therefore, the number of elementary particles or deformations in it in the process of development is a variable quantity varying from $\mathrm{h}$ to he. Its integral in these limits is $\mathrm{jin}=\ln (\mathrm{h} / \mathrm{he})$. It takes time $\tau$ e to detach one particle (that is, the deformation $\Delta$ he he), and $\tau$ (durability) spends on the collapse of the entire system. Then, by proportion, the notation is also true: $\mathrm{jin}=\ln (\tau \mathrm{n} / \tau \mathrm{e})$.

The value of jin determines the number of possible associations (conditionally-of the genus) of strongly connected (nearby) particles. Subsystems of the first order, of size he.1 $>$ he. The size of the genus he. 1 also depends on the pressure, varying from $h$ to he.1. The clan serves as an elementary part of the subsystem of the 2nd order ("tribe"). The integral deformation characterizing the level of interaction of the clans in the tribe is jin. $1=\ln j \mathrm{jin}=\ln (\ln \mathrm{h} / \mathrm{he})$, etc. As the level increases, the elementary particle increases, but the number and strength of bonds in this subsystem are reduced. As a rule, at the $3^{\text {rd }}$ or $4^{\text {th }}$ level, jin.i is close to 1 , and $\ln$ jin.i is close to 0 .

The number of levels (logarithms) to jin.i $\approx 1$ corresponds to the number of previous generations. The reverse action - potentiation, determines the probable number of elementary parts in subsystems of different rank, or their durability. The minimum subsystem"family" includes e $\approx 2.71$ units. Certainty (linearity) is inherent 
only in individual fragments of the world, limited by the "horizon of visibility" (scientific knowledge, common sense, behind which intuition and faith begin) composed of a small number of elements when the integral and derivative deformations are approximately equal: $\ln (\mathrm{h} / \mathrm{he})(\mathrm{h}-\mathrm{he}) /$ he.

\section{Acknowledgments}

None.

\section{Conflicts of interest}

The authors declare that there are no conflicts of interest.

Funding

None. 